\title{
Breastfeeding skills of full-term newborns and associated factors in a low-and-middle-income setting
}

\author{
Esedra E Krüger, Alta AM Kritzinger, Lidia L Pottas
}

Department of Speech-Language Pathology and Audiology, University of Pretoria, South Africa.

\author{
Emails: \\ esedra.kruger@up.ac.za; alta.kritzinger@up.ac.za; lidia.pottas@up.ac.za
}

\begin{abstract}
Background: Normative information on the breastfeeding of term newborns may guide clinicians in early identification of breastfeeding difficulties and oro-pharyngeal dysphagia (OPD), and may support optimal breastfeeding practices.

Objective: To describe breastfeeding skills of term newborn infants in a South African hospital, a lower-middle-income setting, and investigate associations between infants' feeding and other factors.

Method: One breastfeeding session of each of the 71 healthy newborn full-term infants (mean chronological age $=1.9$ days; mean gestation=39.1 weeks) was evaluated using the Preterm Infant Breastfeeding Behavior Scale (PIBBS), suitable for use with term newborns.

Results: All participants were exclusively breastfed. Thirteen participants (18\%) were HIV-exposed. There was no significant difference in the findings of the PIBBS between HIV-exposed and unexposed participants. Most newborns had obvious rooting, latched deeply onto the nipple and some of the areola, had repeated long sucking bursts (mean length=16.82 sucks/burst), and swallowed repeatedly. Most participants were in either the drowsy or quiet-alert state, which are optimal behavioural states for breastfeeding. One to two-hourly on-demand feeds was significantly associated with mothers who had normal births and did not use galactogogues to promote lactation.
\end{abstract}

Conclusion: Results may be used for early identification of OPD in newborns. The findings may be useful to primary care clinicians.

Keywords: Full-term, newborn, breastfeeding, feeding skills, feeding characteristics, normative data.

DOI: https://dx.doi.org/10.4314/ahs.v19i3.43

Cite as: Krüger EE, Kritzinger AAM, Pottas LL. Breastfeeding skills of full-term newborns and associated factors in a low-and-middle-income setting. Afri Health Sci. 2019;19(3): 2670-2678. bttps:/ / dx.doi.org/10.4314/ abs.v19i3.43

\section{Introduction}

Benefits of breastfeeding for all infants have been well-documented ${ }^{1}$. Breastfeeding leads to decreased infant mortality and may reduce otitis media, gastro-intestinal and respiratory infections in children, and has numerous benefits for mothers ${ }^{2}$. In South Africa, a lower middle
Corresponding author:
Esedra Krüger,
Department of Speech-Language
Pathology and Audiology,
Communication Pathology building, corner of Lynnwood Road and Roper Street, Hatfield, Pretoria, South Africa.
Tel: +27124204910
Email: esedra.kruger@up.ac.za

income setting, breastfeeding is a valuable component to infant health and well-being ${ }^{3,4}$. HIV-positive mothers on antiretroviral treatment (ART) have the option to breastfeed exclusively for six months, while their infants receive prophylactic ART ${ }^{5}$. Little is documented about the feeding characteristics of healthy term newborn infants during breastfeeding, especially in this setting.

Descriptions of newborn feeding skills are typically found in textbooks. The rooting reflex is observable and suck-swallow sequences occur approximately once per second while milk is present ${ }^{6}$. Breastfed infants display a higher sucking rate than bottle-fed infants 7 . Ten to 30 suck-swallow-breathe sequences followed by a 3 to 5 second pause constitute a normal sucking burst in breastfeeding infants ${ }^{8}$. Due to limited breastfeeding research, inferences about infants' reactions to bottle feeding have been incorrectly applied to breastfeeding'. Further research is therefore warranted.

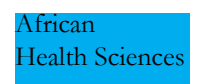

(C) 2019 Krüger et al. Licensee African Health Sciences. This is an Open Access article distributed under the terms of the Creative commons Attribution License (https://creativecommons.org/licenses/BY/4.0), which permits unrestricted use, distribution, and reproduction in any medium, provided the original work is properly cited.

African Health Sciences Vol 19 Issue 3, September, 2019 
Recent breastfeeding research in the term infant population have mainly focused on maternal characteristics during breastfeeding i.e. feeding practices, breastfeeding initiation, breastfeeding rates, perceived breast problems, duration and cessation of breastfeeding, and the introduction of supplementary feeding ${ }^{4,10-13}$. Feeding position, latching-on and sucking in term breastfeeding infants were reported on by Goyal et al. ${ }^{14}$. Hackman et al. ${ }^{15}$ reported on the breastfeeding rates of early term infants. Altuntas et al. ${ }^{16}$ used LATCH scores to compare term infants' breastmilk intake with that of preterm infants. However, research is limited on specific feeding characteristics i.e. rooting, latching on and sucking, of healthy term infants during breastfeeding, especially in contexts where breastfeeding is the most frequently used feeding method. Normative information on the breastfeeding skills of term newborns may guide clinicians in identification of feeding difficulties and oro-pharyngeal dysphagia at the earliest time, and may support optimal breastfeeding practices. Breastfeeding sucking problems are reported as a reason for mothers to not breastfeed exclusively ${ }^{2}$. Further research on the infant's characteristics during breastfeeding is therefore warranted. Breastfeeding difficulties in newborns may be identified and treated timeously while exclusive breastfeeding is promoted. The following research question was posed: What are the breastfeeding skills of term newborns in a South African hospital and are there associations between infants' feeding outcomes and maternal and other factors? The aim of the study was to describe the breastfeeding skills of term newborns and to investigate associations between infants' feeding outcomes and maternal and other factors.

\section{Method}

\section{Study design, setting and population}

In this descriptive cross-sectional study, data were collected prospectively. Ethical clearance was obtained and voluntary participation requested from potential participants' mothers. Data were collected in the postnatal ward of a tertiary hospital that is Baby Friendly Hospital Initiative
(BFHI)-accredited, in Pretoria, South Africa. The ward consists of four open-plan dormitories with eight beds each. Healthy infants room-in with their mothers until discharge home. Mothers are encouraged to breastfeed exclusively on demand as soon as possible from birth. HIV-positive mothers on ART are counselled and given a choice to breastfeed exclusively for six months according to national guidelines 5 . South African hospitals actively encourage exclusive breastfeeding and no formula is provided by state facilities without a prescription and motivation ${ }^{17}$. Mothers of high-risk infants who are unable to breastfeed are given the opportunity to use donor human milk using a cup ${ }^{18}$. HIV-positive mothers with high viral loads are supported to provide infants with pasteurised expressed breast milk using a cup ${ }^{18}$.

Even if mothers intend to bottle feed upon discharge home, bottle feeding is not allowed in the hospital ${ }^{18}$. Purposive sampling was used to recruit 71 infants (mean chronological age $=1.9$ days; standard deviation $[\mathrm{SD}]$ $=0.96)$. All mothers of healthy term newborn infants ( $\leq 4$ days) who were breastfeeding were approached in the postnatal ward. Those with congenital disorders, hyperbilirubinemia, or admitted to intensive care, were excluded. In consultation with a statistician, a sample of 71 infants was deemed appropriate to reach the aim of the study. Gender was evenly distributed (50.7\% male). Infants were born early term $\left(37^{0} / 7\right.$ to $38^{6} / 7$ weeks) or full term $\left(39^{\circ} / 7\right.$ to $\left.41^{6} / 7\right)$ with a mean gestation of 39.1 weeks $(\mathrm{SD}=1.2)$ as determined by the new Ballard score ${ }^{19}$. One infant was born with low birth weight $(2.43 \mathrm{~kg})$, while the mean birth weight of the group was 3.25 kilograms $(\mathrm{SD}=0.5)$. Mothers' mean age was 28.5years $(\mathrm{SD}=6)$. Although the mothers' previous breastfeeding experience was not determined, the majority had more than one child $(73 \%)$ and may therefore have had prior breastfeeding experience. The majority of mothers had South African nationality ( $\mathrm{n}=58 ; 82 \%$ ), while the remaining mothers originated from Zimbabwe, Malawi, and India. Thirteen mothers $(18 \%)$ were HIV positive. Further characteristics are shown in Table 1. 
Table 1. Participant characteristics $(n=71)$

$\begin{array}{ll}\text { Infant characteristics } & \mathbf{n}(\%) \\ \text { Early term } & 23(32) \\ \text { Term } & 48(68) \\ \text { Mode of delivery } & \\ \text { Normal vaginal } & 33(47) \\ \text { Assisted vaginal } & 3(4) \\ \text { Caesarean } & 35(49) \\ \text { Nevirapine received } & 12(17) \\ \text { Maternal characteristics } & \\ \text { Primigravida } & \\ \text { Gravida } \geq 2 & 16(23) \\ \text { Primiparity } & 55(77) \\ \text { Parity } \geq 2 & 19(27) \\ \text { Marital status } & 52(73) \\ \text { Single } & \\ \text { Married } & 51(72) \\ \text { Antenatal care } & 20(28) \\ 0-3 v i s i t s \\ \text { 4visits } \\ \geq 5 \text { visits } & \\ \text { HIV+ } & 22(31) \\ \text { ART } & 21(30) \\ & 28(39) \\ & 13(18) \\ & 12(17)\end{array}$

\section{Data collection}

One feeding session of each participant was clinically evaluated by one speech-language therapist (SLT) experienced in the assessment of feeding and swallowing in neonates, and registered with the Health Professions Council of South Africa (HPCSA). Participants' breastfeeding behaviour was documented using the Preterm Infant Breastfeeding Behavior Scale (PIBBS) ${ }^{20}$. The PIBBS, although called a preterm infant scale, has been developed to also be used with term infants ${ }^{9,21-23}$. The tool has acceptable interrater reliability (agreement $72-92 \%$ ) with an acceptable Kappa score between clinicians $(0.78)^{24}$. The PIBBS was found to have excellent convergent validity in one study ${ }^{24,25}$ and adequate discriminant validity in differentiating gestational age groups ${ }^{20,24}$. The scale's aim is to give mothers suggestions to improve infants' latching-on, sucking, swallowing, and infant state'. The scale, a tool for clinicians and mothers, also documents whether mothers perceive the letdown reflex, and breast-related problems $^{9,20}$. The tool requires mothers to be literate in
English. Most mothers in the current study were not first language English speakers. The PIBBS was therefore completed by a single researcher, an experienced SLT, to increase reliability of the findings. The tool was completed during direct observation of a typical breastfeeding session, either during the morning or the afternoon, according to recommendations by Nyqvist et al. ${ }^{20}$. The SLT stood close enough to the mother to have a clear view of the infant's face and mouth in order to evaluate rooting, latching-on, sucking, longest sucking burst, swallowing and behavioural state ${ }^{20}$. The SLT ensured to observe the feeding session without interference or disturbance. Participants' hospital files were perused, and mothers briefly interviewed for additional demographic, prenatal and feeding information.

\section{Statistical analysis}

The PIBBS $^{20}$ measures breastfeeding characteristics using ratio- and interval scales and findings were compared to typical newborn breastfeeding information. Statistical 
procedures were performed on SAS® version 9.3 (SAS Institute Inc, Carey, NC). Continuous variables were summarised by mean, standard deviation, median, interquartile range, minimum and maximum values. Categorical variables were summarised by frequency counts and percentage calculations. To determine associations between feeding outcomes and demographic characteristics the t-test, Wilcoxon signed-rank test, Fisher's exact test and analysis of variance (ANOVA) were used with a significance level of $\mathrm{p} \leq 0.05$.

\section{Results}

Thirteen participants in this study (18\%) were exposed to HIV. HIV-exposed participants' breastfeeding character- istics according to the $\mathrm{PIBBS}^{20}$ in comparison with that of the unexposed participants are presented in Table 2. Since no statistically significant differences were found between the HIV-exposed and unexposed newborns using the PIBBS, the rest of the results includes the entire sample.

According to the PIBBS, obvious rooting ( $\mathrm{n}=10 ; 77 \%$ ), deep latching $(\mathrm{n}=9 ; 69 \%)$, repeated swallowing ( $n=18 ; 100 \%$ ) were observed in the HIV-exposed group (Table 2). Sucking was characterised by repeated short or long bursts. HIV-exposed participants showed optimal behaviours for breastfeeding.

Most participants in the sample used obvious rooting $(\mathrm{n}=52 ; 73 \%)$ [table 2] and deep latching onto the nipple and some of the areola $(n=56 ; 79 \%)$.

Table 2. Breastfeeding skills using the PIBBS $(n=71)$

Behaviour

Rooting

Some rooting

Obvious rooting

How much of the breast was inside the mouth

Part of nipple

Whole nipple, no part of areola

Nipple and some areola

Sucking

Single sucks, occasional short bursts

Repeated short bursts, occasional long bursts

Repeated long bursts

Swallow

Occasional

Repeated

HIV-unexposed
$\mathbf{n}=\mathbf{5 8}$
$\mathbf{n}(\%)$

$16(28)$

$42(72)$

1(1)

10(17)

$47(81)$

3(4)

19(33)

$36(62)$

1(1)

$57(80)$

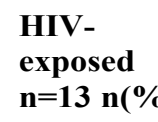

$3(23)$

10(77)

0

$4(31)$

9(69)

0

$7(54)$

6(46)

0

$13(100)$

Total
$\mathbf{n = 7 1}$
$\mathbf{n}(\%)$

1.0000

$32(45)$

$52(73)$

$56(79)$

0.2740

3(4)

26(37)

42(59)

1(1)

70(99)

\section{Sucking}

The majority used repeated long sucking bursts (59\%). Participants' longest sucking burst is displayed in Table 3. The mean length of participants' sucking bursts was 16.82 sucks per burst (Table 3). The longest sucking burst recorded on the PIBBS was indicated as 30 because of the scale's limit being 30+. Therefore, the mean length of the burst may have even been more than represented in Table 3. The minimum and maximum values indicate a large variation, while the IQR shows a normal distribution. 
Table 3. Participants' longest sucking burst (n=71)

$\begin{array}{llll}\text { Behaviour } & \text { Mean(SD) } & \text { Median(IQR) } & \text { Min/Max } \\ \begin{array}{l}\text { Longest sucking } \\ \text { burst (number of } \\ \text { sucks/burst) }\end{array} & 16.82(8.02) & 16(9-24) & 5 / 30 \\ & & & \end{array}$

$\mathrm{IQR}=$ Inter-quartile range, Min=Minimum, Max=Maximum

\section{Behaviour}

Most participants were in either the drowsy $(n=34 ; 48 \%)$ or quiet-alert state $(n=33 ; 47 \%)$, which is ideal for breastfeeding (Figure 1).
The majority of participants ( $\mathrm{n}=46 ; 65 \%$ ) were mostly alert during feeding. Participants' arousal and irritability are shown in Table 4.

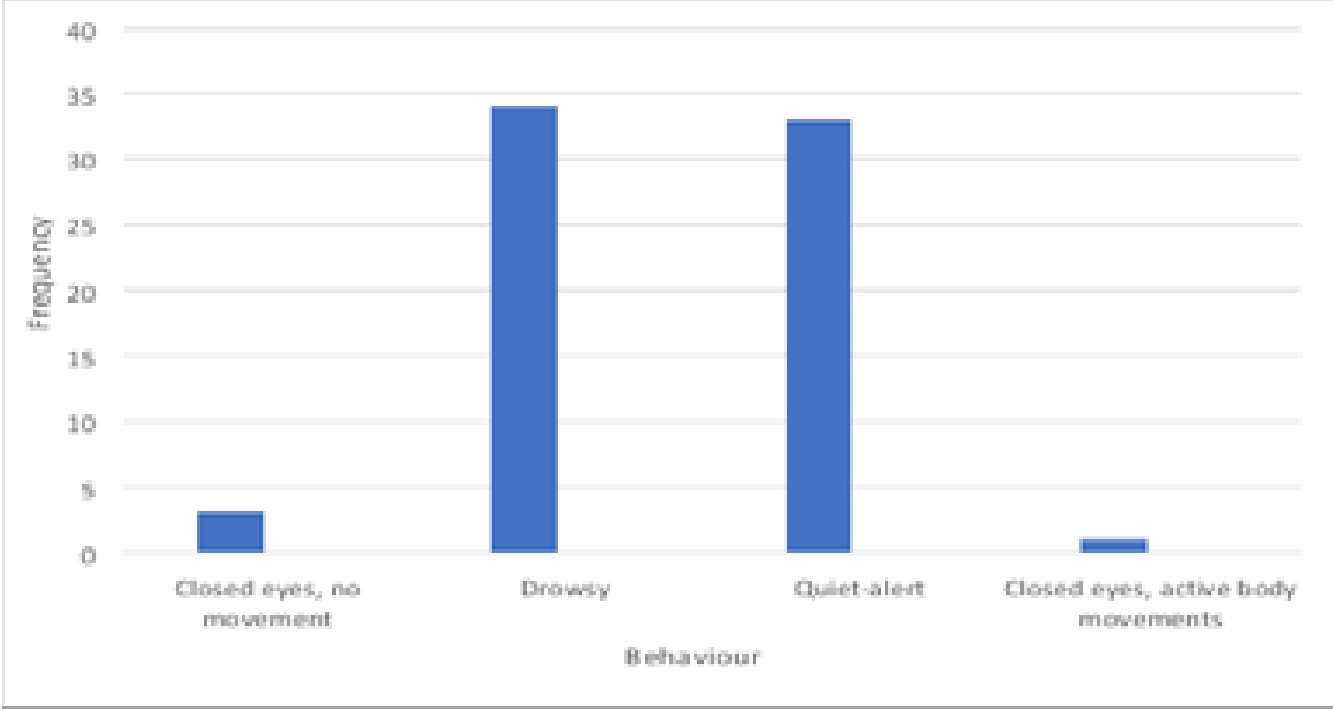

Figure 1. Infant behaviour according to the PIBBS $(n=71)$

Table 4. Arousal and irritability during feeding $(n=71)$

$\begin{array}{ll}\begin{array}{l}\text { Characteristic } \\ \text { Arousal }\end{array} & \mathbf{n}(\%) \\ \text { Sustained 10minutes } & 48(68) \\ \text { Intermittent/fluctuating } & 8(11) \\ \text { Asleep within 4-5minutes } & 15(21) \\ \text { Irritability } & \\ \text { Quiet } & 43(61) \\ \text { Infrequent/quiets easily } & 25(35) \\ \text { Frequent/calms with } & 2(3) \\ \text { holding } & \\ \text { Difficult to quiet } & 1(1)\end{array}$




\section{Maternal and other infant factors}

According to the PIBBS, the majority of mothers ( $n=63 ; 89 \%$ ) experienced the letdown reflex during feeding and reported no breast problems $(n=60 ; 85 \%)$, while pain was experienced in nine mothers $(13 \%)$, and breast swelling in only two mothers $(3 \%)$. Nine mothers $(13 \%)$ were prescribed a galactogogue.

Associations between participants' feeding characteristics and factors related to maternal and infant history were also analysed. Ideal on-demand breastfeeding every one to two hours was associated with normal vaginal delivery $(p=0.044)$ and mothers not using a galactogogue $(p=0.019)$, while having no breast problems were associated with HIV-negative mothers $(p=0.05)$. The higher the Apgar score, the more obvious rooting responses were observed $(\mathrm{p}=0.039)$.

The following observations were made using well-known literature on pediatric dysphagia ${ }^{6,26}$. The entire sample (mean age $=1.9$ days) was exclusively and directly breast fed from birth. All participants presented with normal tone, oral-facial anatomy, and respiration. No coughing, choking, noisy breathing, gagging or emesis was observed. The only distress signs were flaring nostrils ( $\mathrm{n}=1 ; 1.4 \%$ ), and premature fatigue $(\mathrm{n}=10 ; 14 \%)$ based on maternal report. Most participants displayed a good appetite (75\%) as reported by their mothers. All participants were positioned for breastfeeding cradled in the mother's arm. Mothers sat on their beds with their legs out-stretched or hanging down, while some chose to sit on wooden benches mainly used for visitors. The majority of participants were fed on demand one to two-hourly (86\%), while few infants had to be woken three-hourly to feed $(\mathrm{n}=7 ; 10 \%)$. Most participants $(79 \%)$ completed feeds in 20 minutes, while $21 \%(n=15)$ fed for an average of 30 to 40 minutes.

\section{Discussion}

The study provides a valuable description of the breastfeeding characteristics of typical full-term newborns at a South African hospital. The findings are presented from the perspective of the SLT whose role it is to assess the sucking and swallowing of infants in neonatal care if concerns for oropharyngeal dysphagia (OPD) should arise ${ }^{27}$. The value of the findings is that it provides SLTs with quantifiable data on typical infant breastfeeding skills, with maternal breastfeeding practices being less of the focus. The data may serve as preliminary normative information for the PIBBS in the context of a lower-mid- dle-income country such as South Africa, where many high risk infants have breastfeeding difficulties. Preliminary South African normative data for the PIBBS may assist SLTs to easily identify populations requiring early intervention and additional breastfeeding support.

All newborns in the sample were exclusively breastfed from birth, which compares well to the $76.2 \%$ of infants that received exclusive breastfeeding in a study on nursing mothers' feeding practices in India ${ }^{28}$. In 2016 only $32 \%$ of South African infants under six months were breastfed exclusively ${ }^{29}$. Exclusive breastfeeding may further have been encouraged by both nursing staff and the close contact with other mothers in the open plan layout of the ward.

A noteworthy finding was that $18 \%$ of the mothers were HIV positive. According to Zuma et al. ${ }^{30}$ between 2008 and 2012, the peak HIV prevalence in women in South Africa shifted from 25-29y to 30-34y and was $>30 \%$ in 2012. This is higher than found in the current study. Infants born preterm and with health concerns i.e. hyperbilirubinemia, were excluded from the current study, possibly excluding infants born to mothers who are HIV-positive. The association between HIV-negative mothers and no breast problems in our study may be a reminder of the breastfeeding difficulties that HIV-positive mothers may experience. HIV-positive women on ART may present with a wide variety of breast disease ${ }^{31}$, which may explain reports of breast pain and swelling in some mothers.

It is promising that all newborns in the sample were provided with exclusive breastfeeding. South African national guidelines for the prevention of mother to child transmission of $\mathrm{HIV}^{5}$ afford mothers the choice to exclusively breastfeed for six months provided that they use ART, and infants receive prophylactic Nevirapine. Although it is unknown whether this group of 13 infants were HIV-infected, the HIV-exposed newborns did not have breastfeeding characteristics that differed significantly from the unexposed group. Considering the subtle neuro-developmental differences that infants with HIV-exposure may have ${ }^{32}$, it is imperative that this population's feeding abilities be further explored.

Another important finding was that, although most newborns were displaying typical breastfeeding behaviours, and most findings indicated a normal distribution of behaviours, slight variation was found in each characteristic that was documented. There may be some variation in sucking patterns from day to day as well as across feed- 
ing sessions within the first few days after birth ${ }^{33}$. Infants' temperament and the mothers' previous breastfeeding experience may be contributing to this variation.

In this sample only ten infants fell asleep at the breast during feeding. Drowsiness at the breast in term infants is related to the typical release of metabolic hormones such as cholecystokinin during breastfeeding ${ }^{7}$, and could therefore be viewed as normal behaviour instead of distress. Most infants' state of arousal was alert or drowsy, which is in accordance with previous findings of typical breastfeeding. Both are optimal states for feeding ${ }^{6,8,26}$.

The cradle position was used by all mothers. The cradle hold, although being a difficult position for some mothers initially ${ }^{7}$, is most probably the main position encouraged by the hospital staff at this site.

The frequency of feeds was demand-feeding, one to two-hourly, for most infants in the sample, which is encouraging. Supporting unrestricted breastfeeding is one of the ten steps to successful breastfeeding proposed by the World Health Organisation and the United Nations International Children's Fund ${ }^{7,34}$. Infants fed more than seven times per 24 hours show increased milk intake and weight gain compared with those fed less frequent$\mathrm{ly}^{12}$. Only seven infants in the sample had to be woken three-hourly to feed, but that still resulted in eight feeds per 24 hours, and is therefore not a concern.

Notably, one to two-hourly on-demand feeds was significantly associated with mothers who had normal vaginal births and did not have to use galactogogues to promote lactation. Infants delivered using vacuum extraction present with problematic suckling, their mothers often have delayed lactogenesis, and it is associated with early breastfeeding cessation ${ }^{7,26}$. Although no association between birth by caesarean section and feeding was found, caesarean birth is a known breastfeeding hindrance as mothers often have lower levels of prolactin compared to mothers who have a natural birth ${ }^{7}$, which may further explain successful on-demand feeding being associated with normal deliveries. The mothers not using a galactogogue, were more likely to feed infants on-demand at one to two-hourly intervals. Frequent sucking at the breast during the first days after birth will increase the number and sensitivity of prolactin receptors in milk secreting cells that sets the scene for profuse breastmilk production $^{7}$ and therefore galactogogues will not be necessary. Furthermore, only nine mothers in the sample required a galactogogue. Medications are often used to increase milk production, but evidence of effectiveness is limited ${ }^{7}$.

The majority of the sample completed feeds in 20 minutes while some infants $(21 \%)$ fed for an average of 30 to 40 minutes. This finding of prolonged feeding may be related to non-nutritive sucking during the breastfeeding session and increased mother-infant interaction in between nutritive sucking during breastfeeding ${ }^{26}$. Breastfeeding infants usually empty one breast within only four minutes and may continue sucking for pleasure thereafter $^{6}$, which is a shorter duration than the findings in this study.

According to the PIBBS findings, the majority of the sample had obvious rooting, latched deeply onto the nipple and some of the areola, presented with repeated long sucking bursts, and repeated swallowing. Typical full-term newborns demonstrate spontaneous rooting and sucking when brought to the mother's chest ${ }^{7}$, which was found in the majority of this sample. A diminished rooting reflex may have no functional implication for feeding ${ }^{26}$. Research reports a mean of seven to eight sucks per sucking burst in healthy term infants ${ }^{26}$. The mean longest sucking burst in this study $(16.82 ; \mathrm{SD}=8.02)$ was longer than reported in literature. Short sucking bursts lead to inefficient breastfeeding and is related to breastfeeding difficulties ${ }^{7,26}$. Infants with high five-minute Apgar scores were more likely to show obvious rooting for breastfeeding. Low five-minute Apgar scores are associated with increased risk of neurological difficulties in term infants, and successful breastfeeding requires an intact central nervous system ${ }^{23,35}$.

The majority of mothers experienced the letdown reflex during feeding and reported no breast problems. Only nine mothers reported breast pain. Shallow latching onto only the nipple could lead to painful, ineffective feeding ${ }^{36}$. In this study most infants were able to latch deeply.

A limitation of the study is the fact that only one breastfeeding session was observed for each participant and it is known that there might be variability between feeding sessions in one infant. Although a small sample and purposive sampling was utilised, the findings may still be used to identify problematic feeding and possible OPD in the term high-risk newborn population in order to provide early intervention. Further longitudinal research on breastfeeding progress throughout the neonatal stage in this setting may be valuable. 


\section{Conclusion}

A description of typical term newborn breastfeeding skills in a hospital from a lower-middle-income country is provided. Eighteen percent of the sample were exposed to HIV, but did not have significantly different breastfeeding characteristics than the unexposed newborns. The feeding of the HIV-exposed newborn population requires further research as difficulties may emerge over time. The majority of the sample showed optimal breastfeeding behaviours namely obvious rooting, deep latching onto the nipple and some of the areola, longer sucking bursts (mean=16.82; SD=8.02; median=16; IQR=9-24) than reported in literature, and swallowed repeatedly. These findings may be regarded as preliminary normative data for South African newborns for the PIBBS. Consequently, breastfeeding of infants with conditions of high prevalence in South Africa such as hypoxic-ischemic encephalopathy may be evaluated against these findings. The results may promote the BFHI, as it may be used for the early identification of difficulties, which could then be appropriately addressed to support exclusive breastfeeding.

\section{Acknowledgments}

The authors would like to acknowledge the Vice Chancellor's Academic Development Grant, University of Pretoria, South Africa, the Department of Paediatrics, University of Pretoria, as well as the mothers and infants who participated in the study.

\section{Conflict of interest}

None declared.

\section{References}

1. Victoria CG, Bahl R, Barros AJD, Franca GVA, Horton S, Krasavec J, Murch S, Jeeva Sankar M, Walker N, Rollins NC. Breastfeeding in the 21st century: Epidemiology, mechanisms and lifelong effect. Lancet. 2016;387:475-90. 2. Kavle JA, LaCroix E, Dau H, Engmann C. Addressing barriers to exclusive breast-feeding in low- and middle-income countries: A systematic review and programmatic implications. Public Health Nutrition. 2017; 20(17): 312034. DOI: $10.1017 /$ S1368980017002531.

3. Goga AE, Doherty T, Jackson DJ. Infant feeding practices at routine PMTCT sites, South Africa: Results of a prospective observational study amongst HIV exposed and unexposed infants - birth to 9 months. Int Breastfeed J. 2012; 7(4):1-11. DOI: 10.1186/1746-4358-7-4.

4. Ogwu A, Moyo S, Powis K, Asmelash A, Lockman S, Moffat C, Leidner J, Makhema J, Essex M, Shapiro R. Predictors of early breastfeeding cessation among HIV-infected women in Botswana. Trop Med Int Health. 2016;21(8):1013-8, DOI: 10.1111/tmi.12729.

5. National Department of Health. National consolidated guidelines for the prevention of mother-to-child transmission of HIV and management of HIV in children, adolescents and adults. Pretoria: National Department of Health; 2015.

6. Arvedson JC, Brodsky L. Pediatric swallowing and feeding. Assessment and management 2nd Ed. Albany, New York: Thomson Delmar Learning; 2002.

7. Walker M. Breastfeeding management for the clinician. Using the evidence $4^{\text {th }}$ Ed. Burlington: Jones \& Bartlett Learning; 2017.

8. Genna CW, Sandora L. Breastfeeding: Normal sucking and swallowing. In CW Genna, Supporting sucking skills in breastfeeding infants $2^{\text {nd }} \mathrm{Ed}$. Burlington: Jones \& Bartlett Learning; 2013.

9. Nyqvist K. Breastfeeding preterm infants. (2013). Supporting sucking skills in breastfeeding infants $2^{\text {nd }} \mathrm{Ed}$. Burlington: Jones \& Bartlett Learning; 2013.

10. Doherty T, Sanders D, Jackson D, Swanevelder S, Lombard C, Zembe W, Chopra M, Goga A, Colvin M, Fadnes LT, Engebretsen IMS, Ekstrom E, Tylleskar T. Early cessation of breastfeeding amongst mothers in South Africa: An area needing urgent attention to improve child health. BMC Pediatr. 2012;12(105):1-10. DOI:10.1186/1471-2431-12-105.

11. Nevo N, Rubin L, Tamir A, Levine A, Shaoul R. Infant feeding patterns in the first 6 months: An assessment in full-term infants. J Pediatr Gastroenterol. 2007;45:234-9.

12. Yamauchi Y, Yamanuchi I. Breast-feeding frequency during the first 24 hours after birth in full-term neonates. Pediatrics. 1990;86(2):171-5.

13. Yilmaz E, Yilmaz Z, Hatice I, Burak GI, Hakan T, Fadil K, Tuncay K. Factors associated with breastfeeding initiation and exclusive breastfeeding rates in Turkish adolescent mothers. Breastfeed Med. 2016;11(6):315-20. DOI: 10.1089/bfm.2016.0012. 2016.

14. Goyal RC, Banginwar AS, Ziyo F, Toweir AA. Breastfeeding practices: Positioning, attachment (latch-on) and effective suckling - A hospital-based study in Libya.J Fam Community Med. 2011;18(2):74-9. 
15. Hackman NM, Alligood-Percoco N, Martin A, Zhu J, Kjerulff KH. Reduced breastfeeding rates in firstborn late preterm and early term infants. Breastfeed Med. 2016;11(3):119-25, DOI: 10.1089/bfm.2015.0122.

16. Altunas N, Kocak M, Akkurt S, Razi HC, Kislal MF. LATCH scores and milk intake in preterm and term infants: A prospective comparative study. Breastfeed Med. 2015;10(2):96-101. DOI: 10.1089/bfm.2014.0042.

17. National Department of Health. Mother Child and Women's Health National Strategic Plan for South Africa 2012-2016. 2012. National Department of Health, South Africa.

18. Van Rooyen E. Infant feeding policy of the Kangaroo Mother Care Unit. Kalafong Hospital, South Africa. 2018. Pretoria, South Africa.

19. Ballard JL, Khoury JC, Wedig K, Wang L, Eilers-Welsman BL, Lipp R. New Ballard score, expanded to include extremely premature infants. J Pediatr. 1991;119(3):41723. DOI: 10.1016/S0022-3476(05)82056-6.

20. Nyqvist KH, Rubertsson C, Ewald U, Sjöden P. Development of the Preterm Infant Breastfeeding Behavior Scale (PIBBS): A study of nurse-mother agreement. J Hum Lact. 1996;12(3):207-19.

21. Da Costa SP, van den Engel-Hoek L, Bos AF. Stateof-the-art. Sucking and swallowing in infants and diagnostic tools. J Perinatol. 2008;28, 247-57.

22. Radzyminski S. The effect of ultra low dose epidural analgesia on newborn breastfeeding behaviors. J Obstet Gynecol Neonatal Nurs. 2003;32(3):322-31.

23. Radzyminski S. Neurobehavioral functioning and breastfeeding behavior in the newborn. $J \mathrm{Ob}$ stet Gynecol Neonatal Nurs. 2005;34(3):335-41, DOI: $10.1177 / 0884217505276283$.

24. Howe TH, Lin KC, Fu CP, Su CT, Hsieh CL. A Review of psychometric properties of feeding assessment tools used in neonates. JOGNN. 2008;37:338-349, DOI: 10.1111/j.152-6909.2008.00240.x

25. Nyquist KH, Farnstrand C, Eeg-Olofsson KE, Ewald $\mathrm{U}$. Early oral behaviour in preterm infants during breastfeeding: An electromyographic study. Acta Paediatrica. 2001;90:658-663.

26. Wolf LS, Glass RP. Feeding and swallowing disorders in infancy. Assessment and management. Tucson: Therapy Skill Builders; 1992.
27. American Speech-Language-Hearing Association (ASHA). Scope of practice in Speech-Language Pathology [Scope of practice]. 2016. Available from www.asha.org/policy. 28. Srinivasa K, Abhishek P, Ajay J, Manjunath GA. Infant feeding practices among nursing mothers at a rural tertiary care hospital. Int J Contemp Pediatr. 2017;4(3):83742, DOI: 10.18203/2349-3291.ijcp20171525.

29. Statistics South Africa. South Africa Demographic and Health Survey. Key indicator report. 2017. Statistics South Africa.

30. Zuma K, Shisana O, Rehle TM, Simbayi LC, Jooste S, Zungu N, Labadarios D, Onoya D, Evans M, Moyo S, Abdullah F. New insights into HIV epidemic in South Africa: Key findings from the National HIV prevalence, incidence and behaviour survey, 2012. Afr J AIDS Res. 2016;15(1):6775, DOI: 10.2989/16085906.2016.1153491.

31. Pantanowitz L, Sen S, Crisi GM, Makari-Judson G, Garb J, Skiest D. Spectrum of breast disease encountered in HIV-positive patients at a community teaching hospital. Breast. 2011;20:303-8, DOI: 10.1016/j.breast.2010.08.00. 32. Le Doaré K, Bland R, Newell ML. Neurodevelopment in children born to HIV-infected mothers by infection and treatment status. Pediatrics. 2012;130:e1326-44. 33. Medoff-Cooper B, Bilker W, Kaplan JM. Sucking and behavioural state in 1- and 2-day old full-term infants. J Obstet Gynaecol Neonatal Nurs. 2010;39:514-29, DOI: 10.1111/j.1552-6909.2010.01173.x.

34. World Health Organization, \& United Nations Children's Fund. Implementation guidance: Protecting, promoting and supporting breastfeeding in facilities providing maternity and newborn services-the revised baby-friendly hospital initiative. 2018.Geneva: World Health Organization. Retrieved from http://apps.who.int/bookorders.

35. Genna CW, LeVan Fram J, Sandora L. Neurological issues and breastfeeding. In CW Genna (Ed.). Supporting sucking skills in breastfeeding infants (2nd Ed.), Burlington: Jones \& Bartlett Learning; 2013.

36. Glover R, Wiessinger D. They can do it, you can help: Building breastfeeding skill and confidence in mother and helper. In CW Genna (Ed.). Supporting sucking skills in breastfeeding infants ( $2^{\text {nd }} E d$.), Burlington: Jones \& Bartlett Learning; 2013. 